\title{
Radar Signal Intra-Pulse Feature Extraction Based on Improved Wavelet Transform Algorithm
}

\author{
Wenxu Zhang, Fuli Sun, Bing Wang \\ College of Information and Communication Engineering, Harbin Engineering University, Harbin, China \\ Email: zhangwenxu@hrbeu.edu.cn,s316087018@hrbeu.edu.cn,wangbing2016@hrbeu.edu.cn
}

How to cite this paper: Zhang, W.X., Sun, F.L. and Wang, B. (2017) Radar Signal IntraPulse Feature Extraction Based on Improved Wavelet Transform Algorithm. Int. J. Communications, Network and System Sciences, 10, 118-127.

https://doi.org/10.4236/ijcns.2017.108B013

Received: May 18, 2017

Accepted: August 11, 2017

Published: August 14, 2017

\begin{abstract}
With the new system radar put into practical use, the characteristics of complex radar signals are changing and developing. The traditional analysis method of one-dimensional transformation domain is no longer applicable to the modern radar signal processing, and it is necessary to seek new methods in the two-dimensional transformation domain. The time-frequency analysis method is the most widely used method in the two-dimensional transformation domain. In this paper, two typical time-frequency analysis methods of short-time Fourier transform and Wigner-Ville distribution are studied by analyzing the time-frequency transform of typical radar reconnaissance linear frequency modulation signal, aiming at the problem of low accuracy and sensitivity to the signal noise of common methods, the improved wavelet transform algorithm was proposed.
\end{abstract}

\section{Keywords}

Intra-Pulse Feature Extraction, Time-Frequency Analysis, Short-Time Fourier Transform, Wigner-Ville Distribution, Wavelet Transform

\section{Introduction}

The characteristics of modern complex radar signals penetrate into the time domain, frequency domain, airspace, two-dimensional transformation domain, which contains rich content; it is reflected in the subtle differences of the signal itself as well as in the characteristics and changes of the overall signal [1]. The traditional feature analysis and extraction methods are mostly reflected in the characteristics of a signal in a certain one-dimensional transformation domain (time domain, frequency domain, airspace), while the characteristics of other two-dimensional transformation domain are not obvious. Since the complex 
signal feature analysis and extraction method should be carried out in the two-dimensional transformation domain, that is, the traditional method fails to extract the complex characteristic parameters of modern radar signals, thus affecting the reconnaissance effect of complex radar signals [2].

The time-frequency analysis method is the most widely used method in the two-dimensional transform domain. The time-frequency analysis method can reflect the time-varying nature of the non-stationary signal more accurately [3], so that the analysis of the non-stationary signal is accurate to the specific frequency of the specific time. There are many kinds of time-frequency analysis methods, among them, Short-time Fourier transform, Wigner-Ville distribution and wavelet transform are widely used in the analysis and feature extraction of complex signals [4].

The literature [5] proposed an adaptive short-time Fourier analysis method, however it didn't study the problem that the time resolution and the frequency resolution cannot be taken into account simultaneously. In the literature [6], the authors proposed a method of multi resolution signal analysis using improved Wigner-Ville distribution. But there is still cross-term interference when using it to analyze multi-component LFM signals, which seriously affects the extraction of the time-frequency characteristics of signal. The literature [7] proposed an improved Morlet wavelet and its complete transform. However, the authors didn't study that how to apply the method in the radar signal intra-pulse feature extraction. Aiming at the problem of low accuracy and sensitivity to the signal noise of common methods in the radar signal intra-pulse feature extraction, this paper proposed the improved wavelet transform algorithm.

The linear frequency modulation (LFM) signal is one of the modern radar signals that are frequently used with a simple structure. It obtains a large time-bandwidth product by the linear frequency modulation. It is a typical signal type that is widely used in pulse compression signals. The LFM signal has the advantage of Doppler shift insensitivity, which means that even if the Doppler shift of the echo signal is large, the matched filter still has the effect of pulse pressure [8]. Typical time-frequency analysis methods of short-time Fourier transform, Wigner-Ville distribution and improved wavelet transform algorithm are studied by analyzing the time-frequency transform of typical radar reconnaissance LFM signal.

\section{Typical Time-Frequency Analysis Methods}

Short-time Fourier transform (STFT) is a linear transformation, which is a typical representation of the current time-frequency analysis. The small calculation and conceptual simplicity make Short-time Fourier transform a widely used time-frequency analysis in engineering [5]. The method of time-frequency distribution adopting bilinear transformation whose basic idea is to design the joint function of time and frequency to describe the energy density or intensity of the signal at different time and frequency [9]. The Wigner-Ville distribution (WVD) is the most typical time-frequency distribution [6]. 


\subsection{Short-Time Fourier Transform}

In order to introduce time-dependency in the Fourier transform, a simple and intuitive solution consists in pre-windowing the signal $x(u)$ around a particular time $t$, calculating its Fourier transform, and doing that for each time instant $t$. The resulting transform, called the short-time Fourier transform (STFT) [10], is

$$
F_{x}(t, v ; h)=\int_{-\infty}^{+\infty} x(u) h *(u-t) e^{-j 2 \pi v u} d u .
$$

where $h(t)$ is a short time analysis window localized around $t=0$ and $v=0$. Because multiplication by the relatively short window $h *(u-t)$ effectively suppresses the signal outside a neighborhood around the analysis time point $u=t$, the STFT is a "local" spectrum of the signal $x(u)$ around $t$.

The STFT may also be expressed in terms of signal and window spectra:

$$
F_{x}(t, v ; h)=\int_{-\infty}^{+\infty} X(\xi) H^{*}(\xi-v) \exp [j 2 \pi(\xi-v) t] d \xi .
$$

where $X$ and $H$ are respectively the Fourier transforms of $x$ and $h$. Thus, the STFT $F_{x}(t, v ; h)$ can be considered as the result of passing the signal $x(u)$ through a band-pass filter whose frequency response is $H^{*}(\xi-v)$, and is therefore deduced from a mother filter $H(\xi)$ by a translation of $v$. So the STFT is similar to a bank of band-pass filters with constant bandwidth [7].

\subsection{Wigner-Ville Distribution}

In contrast with the linear time-frequency representations which decompose the signal on elementary components, the purpose of the energy distributions is to distribute the energy of the signal over the two description variables: time and frequency. The starting point is that since the energy of a signal $x$ can be deduced from the squared modulus of either the signal or its Fourier transform [6],

$$
E_{x}=\int_{-\infty}^{+\infty}|x(t)|^{2} d t=\int_{-\infty}^{+\infty}|X(v)|^{2} d v .
$$

we can interpret $|x(t)|^{2}$ and $|X(v)|^{2}$ as energy densities, respectively in time and in frequency. It is then natural to look for a joint time and frequency energy density $\rho_{x}(t, v)$, such that

$$
E_{x}=\int_{-\infty}^{+\infty} \int_{-\infty}^{+\infty} \rho_{x}(t, v) d t d v .
$$

which is an intermediary situation between those described by (4). As the energy is a quadratic function of the signal, the time-frequency energy distributions will be in general quadratic representations. Two other properties that an energy density should satisfy are the following marginal properties:

$$
\begin{aligned}
& \int_{-\infty}^{+\infty} \rho_{x}(t, v) d t=|X(v)|^{2} . \\
& \int_{-\infty}^{+\infty} \rho_{x}(t, v) d v=|x(t)|^{2} .
\end{aligned}
$$

which mean that if we integrate the time-frequency energy density along one variable, we obtain the energy density corresponding to the other variable.

A time-frequency energy distribution which is particularly interesting is the 
Wigner-Ville distribution (WVD) defined as:

$$
W_{x}(t, v)=\int_{-\infty}^{+\infty} x(t+\tau / 2) x^{*}(t-\tau / 2) e^{-j 2 \pi v \tau} d \tau
$$

or equivalently as:

$$
W_{x}(t, v)=\int_{-\infty}^{+\infty} X(v+\xi / 2) X^{*}(v-\xi / 2) e^{j 2 \pi \xi t} d \xi .
$$

This distribution satisfies a large number of desirable mathematical properties, as summarized in the next sub-section. In particular, the WVD is always real-valued, it preserves time and frequency shifts and satisfies the marginal properties.

\section{Improved Wavelet Analysis}

Wavelet analysis is a kind of time-frequency analysis aiming to describe the signal with time-scale plane based on the idea of replacing the representation of frequency domain with scale field, which has the characteristics of multiresolution analysis together with the ability to indicate the local characteristics of the signal. Wavelet analysis is a localized analysis method of time and frequency in which the size of time-frequency window is fixed but the shape can be adjusted [11].

\subsection{Continuous Wavelet Transform Principle}

The idea of the continuous wavelet transform (CWT) is to project a signal $x$ on a family of zero-mean functions (the wavelets) deduced from an elementary function (the mother wavelet) by translations and dilations:

$$
T_{x}(t, a ; \Psi)=\int_{-\infty}^{+\infty} x(s) \Psi_{t, a}^{*}(s) d s .
$$

where

$$
\Psi_{t, a}(s)=|a|^{-1 / 2} \Psi\left(\frac{s-t}{a}\right) .
$$

The variable $a$ corresponds now to a scale factor, in the sense that taking $|a|>1$ dilates the wavelet $\Psi$ and taking $|a|<1$ compresses $\Psi$.

The basic difference between the wavelet transform and the short-time Fourier transform is as follows: when the scale factor $a$ is changed, the duration and the bandwidth of the wavelet are both changed but its shape remains the same. And in contrast to the STFT, which uses a single analysis window, the CWT uses short windows at high frequencies and long windows at low frequencies. This partially overcomes the resolution limitation of the STFT [11]. The CWT can also be seen as a filter bank analysis composed of band-pass filters with constant relative bandwidth.

\subsection{Morlet Wavelet and Its Improved Algorithm}

Morlet wavelet is chosen as the wavelet basis function to extract the characteristic parameters of radar signals because of its good time-frequency characteristics; its expression is [12]:

$$
m(t)=e^{j \omega_{0} t} e^{-t^{2} / 2}
$$


and its Fourier transforms is:

$$
M(\omega)=\int_{-\infty}^{+\infty} e^{j \omega_{0} t} e^{-t^{2} / 2} e^{-j \omega t} d t=\sqrt{2 \pi} e^{-\left(\omega-\omega_{0}\right)^{2} / 2} .
$$

So:

$$
M(0)=\sqrt{2 \pi} e^{-\omega_{0}^{2} / 2} \neq 0 .
$$

The improved wavelet transform has two purposes: 1) transform the time signal into time-frequency domain instead of time scale domain by means of wavelet transform with kernel function of Morlet wavelet; 2) each parameter of the wavelet has definite physical meaning.

The improved Morlet basic wavelet is as follow:

$$
\psi(t)=\frac{1}{\sqrt{2 \pi}} \exp \left(-\frac{t^{2}}{2}+j 2 \pi t\right), \omega_{0}=2 \pi .
$$

In the formula, $\frac{1}{\sqrt{2 \pi}}$ is the wavelet amplitude parameter, which makes:

$$
\int_{R}|\psi(t)| d t=1
$$

The family of Morlet wavelet deduced from the mother wavelet by translations and dilations:

$$
\psi_{f, \tau}(t)=\frac{|f|}{\sqrt{2 \pi}} \exp \left[-\frac{f^{2}(t-\tau)^{2}}{2}+j 2 \pi f(t-\tau)\right] .
$$

In the formula, the parameters $t$ and $\tau$ are the time, and $f$ is the wavelet dominant frequency.

The wavelet transform using Morlet wavelet as kernel function can be used for time-frequency analysis of signals. Morlet wavelet is a double window function. The time window center $t_{0}$ of $\psi_{f, \tau}(t)$, frequency window center $f_{0}$, time window radius $\Delta t$ and frequency radius $\Delta f$ are:

$$
t_{0}=\tau, f_{0}=f, \Delta t=\frac{1}{\sqrt{2} f}, \Delta f=\frac{f}{2 \sqrt{2} \pi} .
$$

The time-frequency window is:

$$
\left[\tau-\frac{1}{\sqrt{2} f}, \tau+\frac{1}{\sqrt{2} f}\right] \times\left[f-\frac{1}{2 \sqrt{2} \pi}, f+\frac{1}{2 \sqrt{2} \pi}\right] .
$$

and

$$
\Delta t \Delta f=\frac{1}{\sqrt{2} f} \cdot \frac{f}{2 \sqrt{2} \pi}=\frac{1}{4 \pi} .
$$

The modified Morlet wavelet approximation satisfies the admissible conditions, and

$$
\psi_{f, \tau}(t)=0,(f=0) .
$$

The function space composed of the function family generated by the improved Morlet wavelet function is equal to the function space generated by the original Morlet wavelet function family. 


\section{Simulation Experiment and Analysis}

\subsection{Short-Time Fourier Transform Time-Frequency Graphs of LFM Signals with Different SNR}

Figure 1 shows the short-time Fourier transform, and there is no cross-term interference for multi-component LFM signal since STFT belongs to linear transformation, which makes it suitable for detecting linear frequency modulation signal, and it can detect the basic characteristics of the signal even when SNR drops to $-5 \mathrm{~dB}$. However, due to the fixed length of the sliding window, the time resolution and the frequency resolution cannot be taken into account simultaneously, so the accuracy of analysis is relatively low.

\subsection{Wigner-Ville Distribution Time-Frequency Graphs of LFM Signals with Different SNR}

Without going into details about this representation we can see that the linear progression of the frequency with time is clearly shown in Figure 2. WVD has the feature of strong energy aggregation in LFM signals. There is obvious cross-term interference when using it to analyze multi-component LFM signals, which seriously affects the extraction of the time-frequency characteristics of signal, and WVD is very sensitive to noise.

\subsection{Improved Wavelet Transform Time-Frequency Graphs of LFM Signals with Different SNR}

The improved wavelet transform has introduced the scalable parameters in the window function to obtain the automatic resolution, that is, in the part of low frequency obtain the higher frequency resolution and the lower time resolution, and in the part of high frequency obtain the higher time resolution and the lower frequency resolution. Thus it is suitable for detecting the transient anomalies in the normal signal entrainment and shows its details, which is very useful for detecting the trip points of frequency coded and phase coded signal. And the effect of continuous wavelet analysis has not changed considerably with the existence of noise, which indicates that wavelet analysis is not sensitive to noise, as is shown in Figure 3.

\section{Conclusion}

Aiming at the problem of low accuracy and sensitivity to the signal noise of short-time Fourier transform and Wigner-Ville distribution, this paper proposed the improved wavelet transform algorithm. The results of simulation show that the performance of the extraction of the extraction of radar signal features which is based on the improved wavelet transform algorithm better than the others, and the method is feasible. The optimal feature extraction method used for different modulation signals is different. At present, feature extraction methods of complex radar reconnaissance signal proposed by researchers at home and abroad involve signal detection, parameter estimation and classification of modulation style. In the future, the characteristics of different coded modulation 


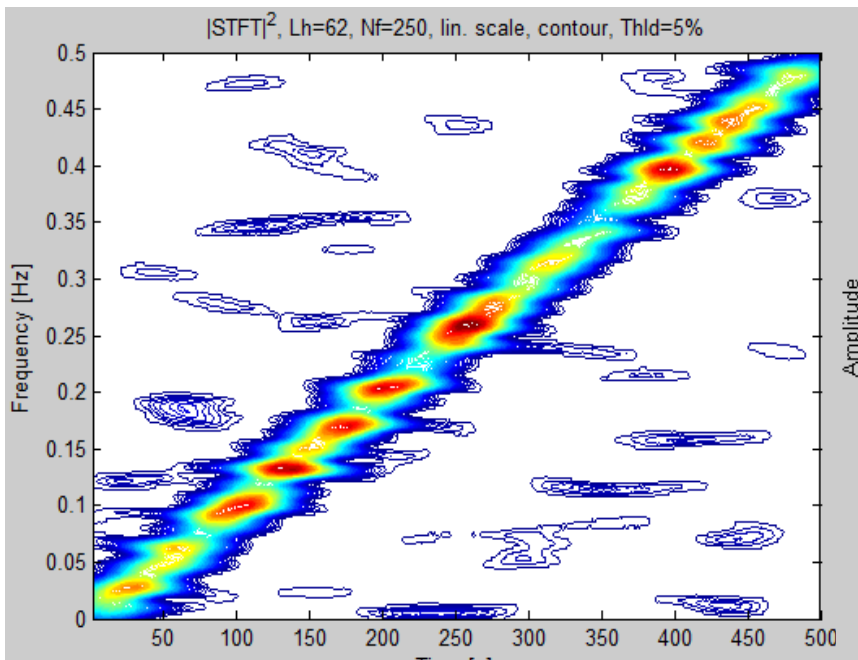

(a)

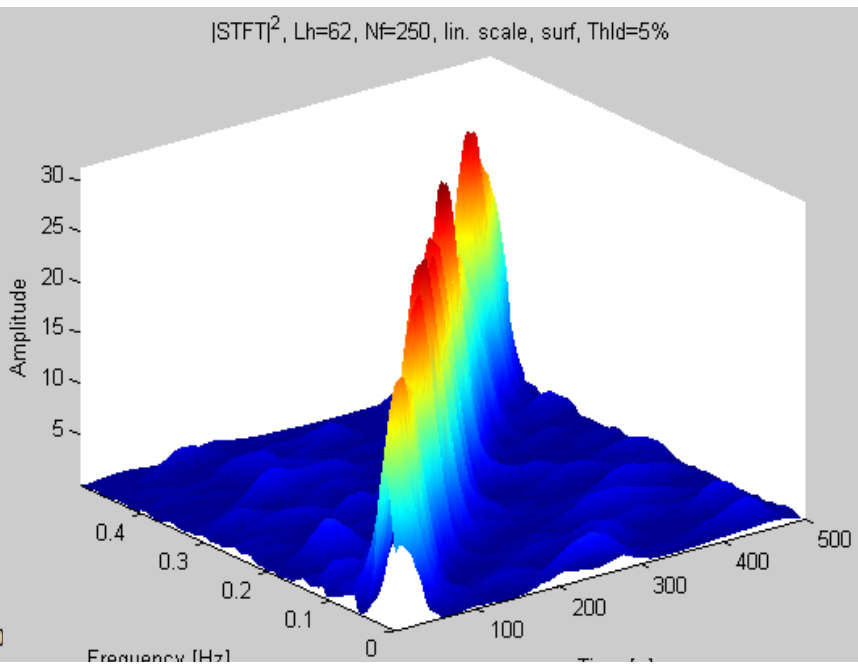

(b)

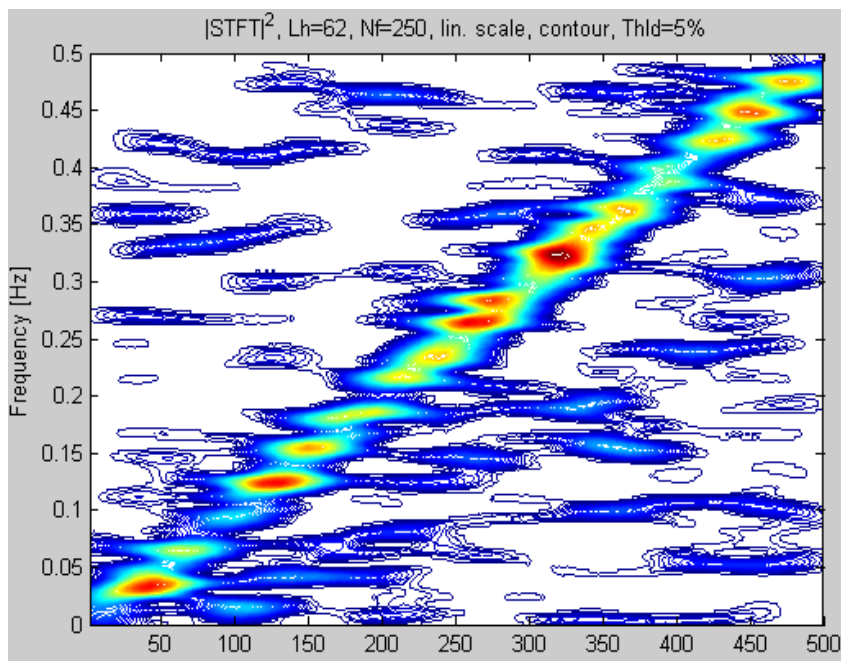

(c)

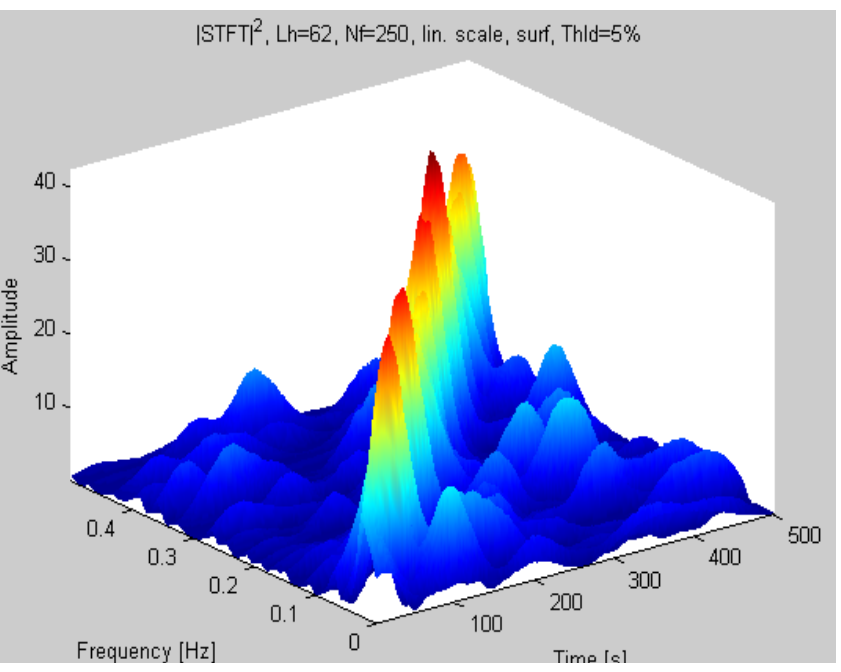

(d)

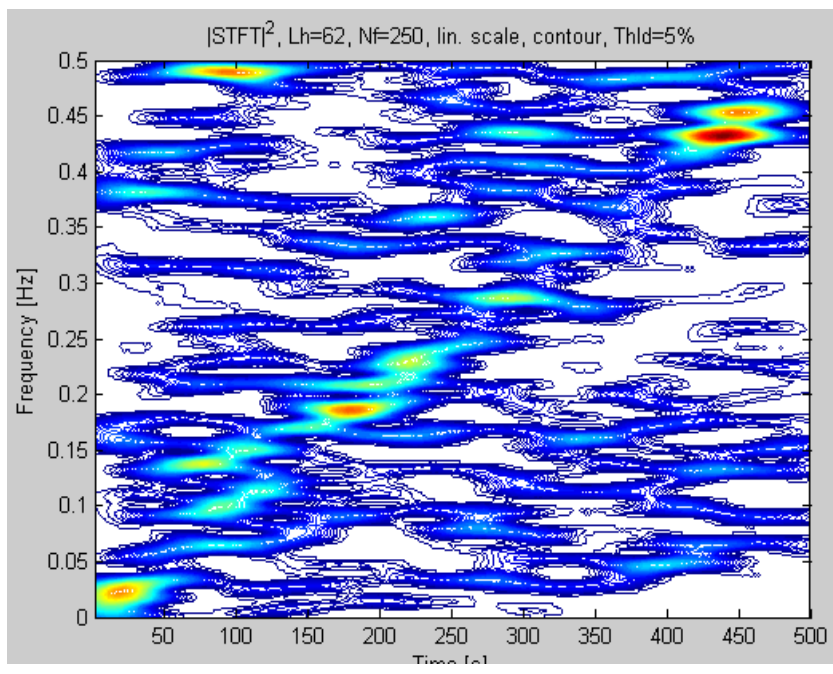

(e)

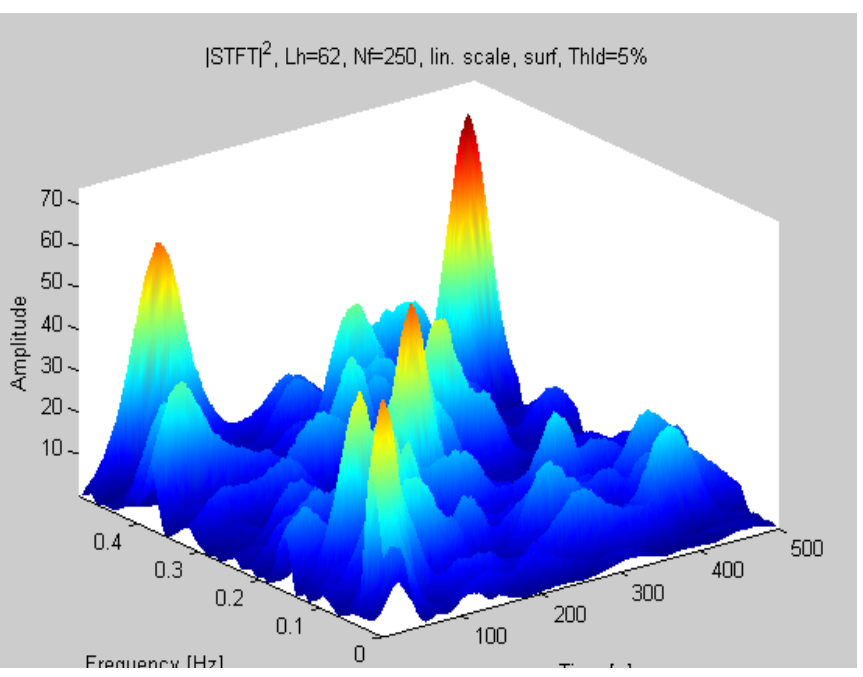

(f)

Figure 1. Short-time Fourier transform time-frequency Graphs of LFM signals. (a) $\mathrm{SNR}=5 \mathrm{~dB}$ (two-dimensional). (b) $\mathrm{SNR}=5$ $\mathrm{dB}$ (three-dimensional). (c) SNR $=0 \mathrm{~dB}$ (two-dimensional). (d) SNR $=0 \mathrm{~dB}$ (three-dimensional). (e) $\mathrm{SNR}=-5 \mathrm{~dB}$ (two-dimensional). (f) $\mathrm{SNR}=-5 \mathrm{~dB}$ (three-dimensional). 


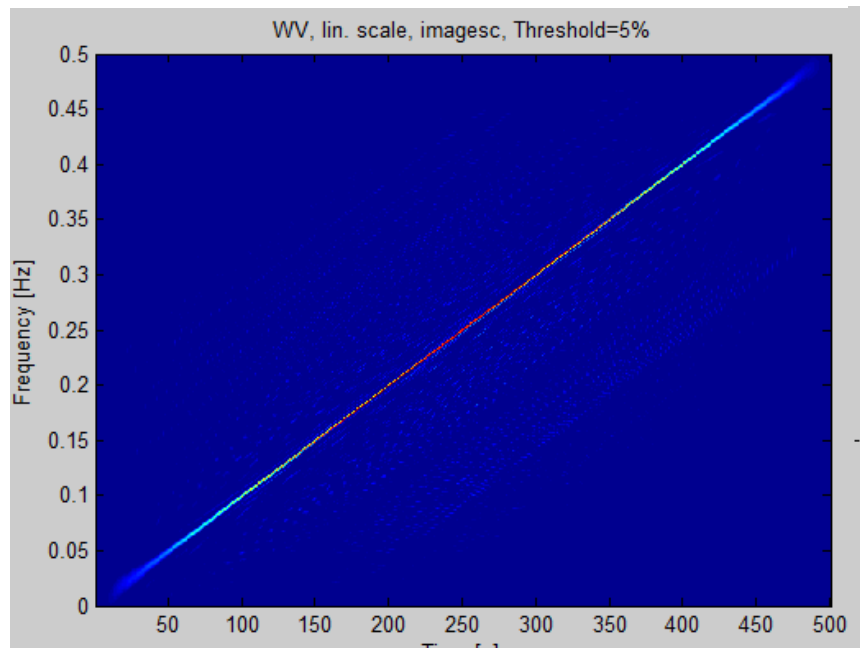

(a)

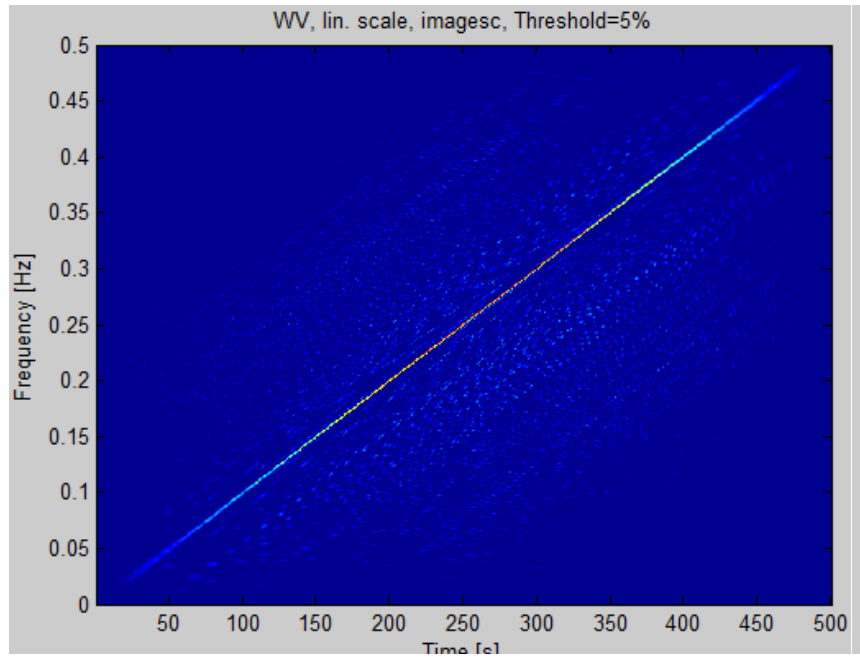

(c)

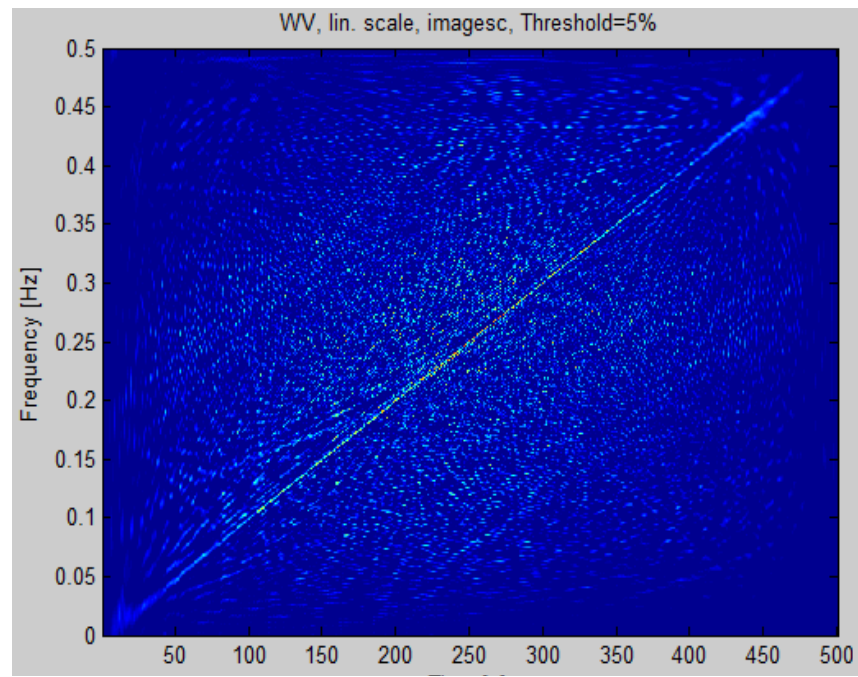

(e)

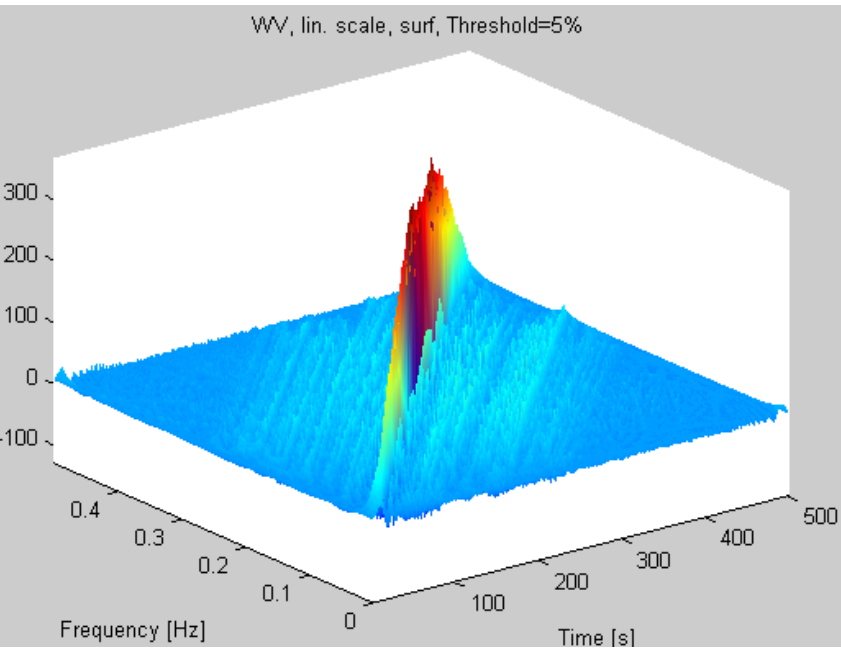

(b)

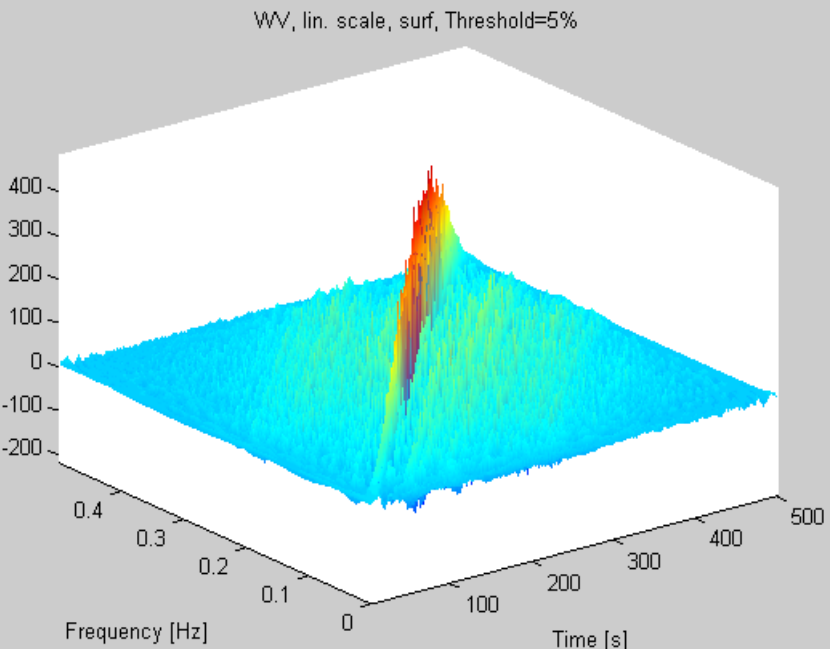

(d)

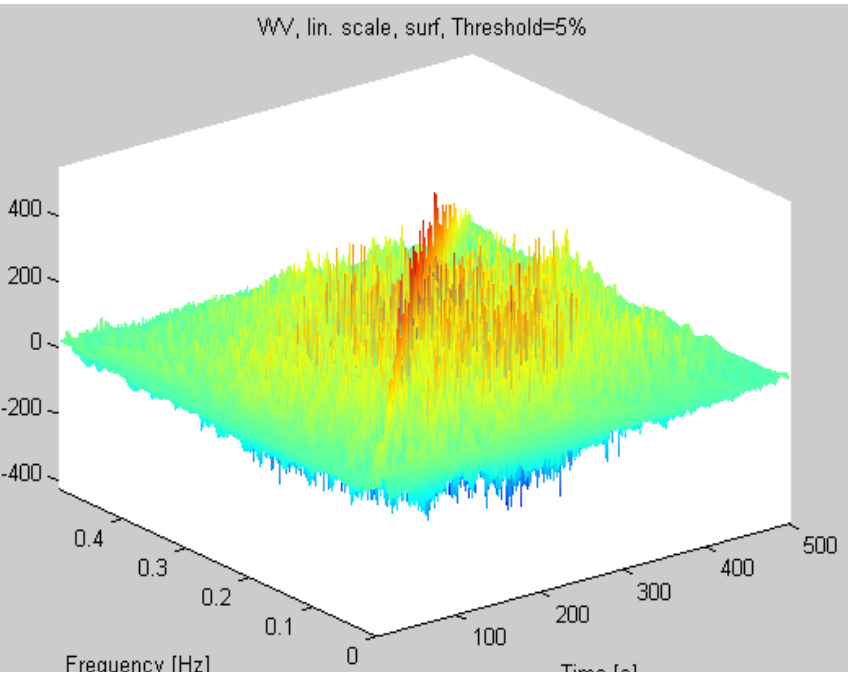

(f)

Figure 2. Wigner-Ville distribution time-frequency Graphs of LFM signals. (a) $\mathrm{SNR}=5 \mathrm{~dB}$ (two-dimensional). (b) $\mathrm{SNR}=5 \mathrm{~dB}$ (three-dimensional). (c) SNR $=0 \mathrm{~dB}$ (two-dimensional). (d) SNR $=0 \mathrm{~dB}$ (three-dimensional). (e) $\mathrm{SNR}=-5 \mathrm{~dB}$ (two-dimensional). (f) $\mathrm{SNR}=-5 \mathrm{~dB}$ (three-dimensional). 

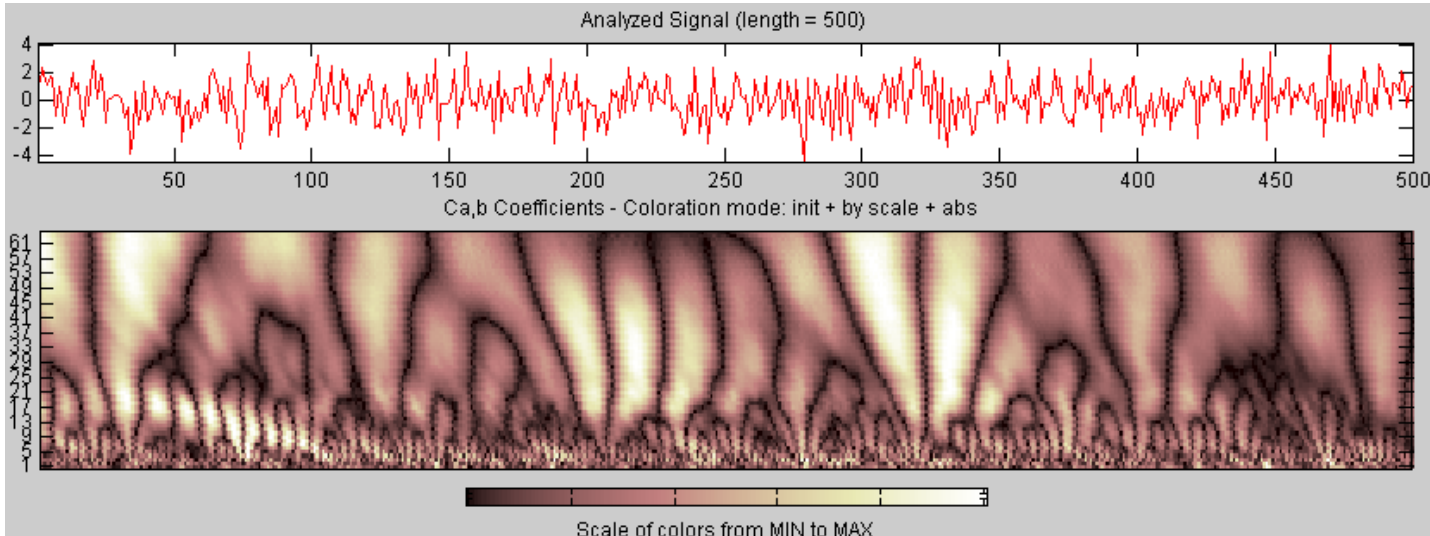

Coefficients Line $-\mathrm{Ca}, \mathrm{b}$ for scale $\mathrm{a}=32$ (frequency $=0.022$ )
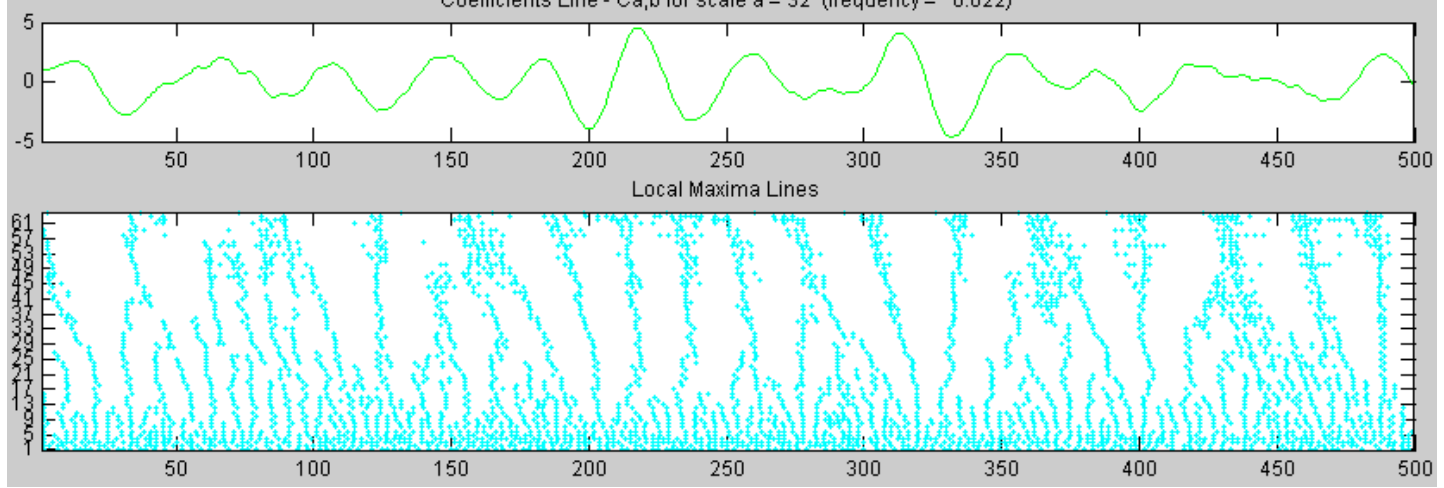

(a)
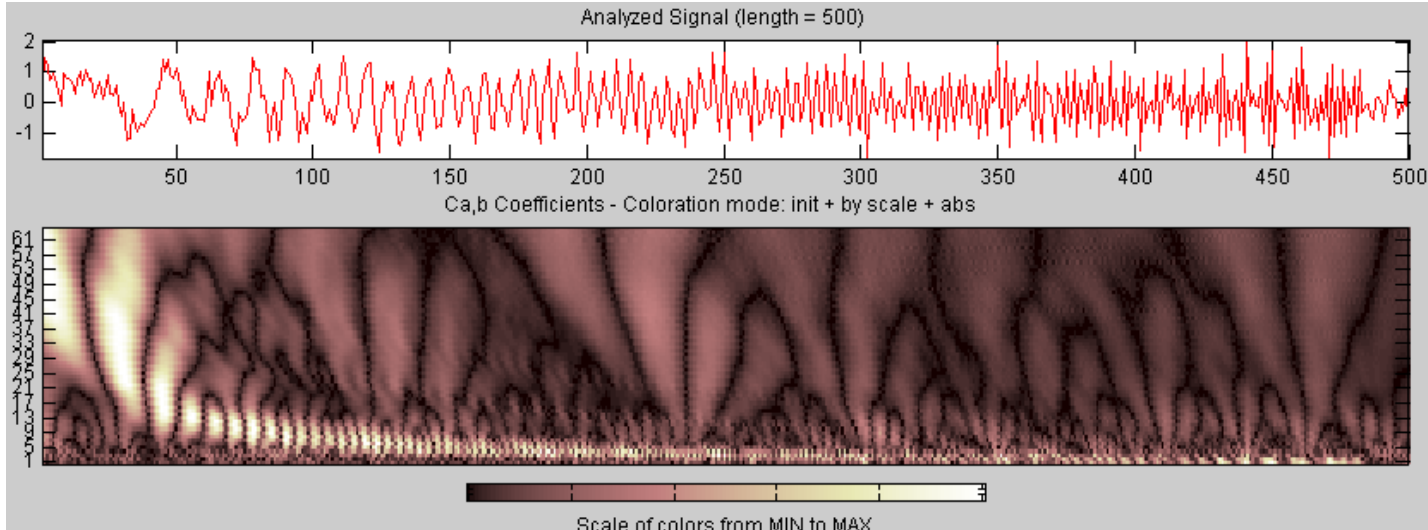

Scale of colors from MIN to MAX

Coefficients Line $-\mathrm{C} a, b$ for scale $a=32$ (frequency $=0.022$ )
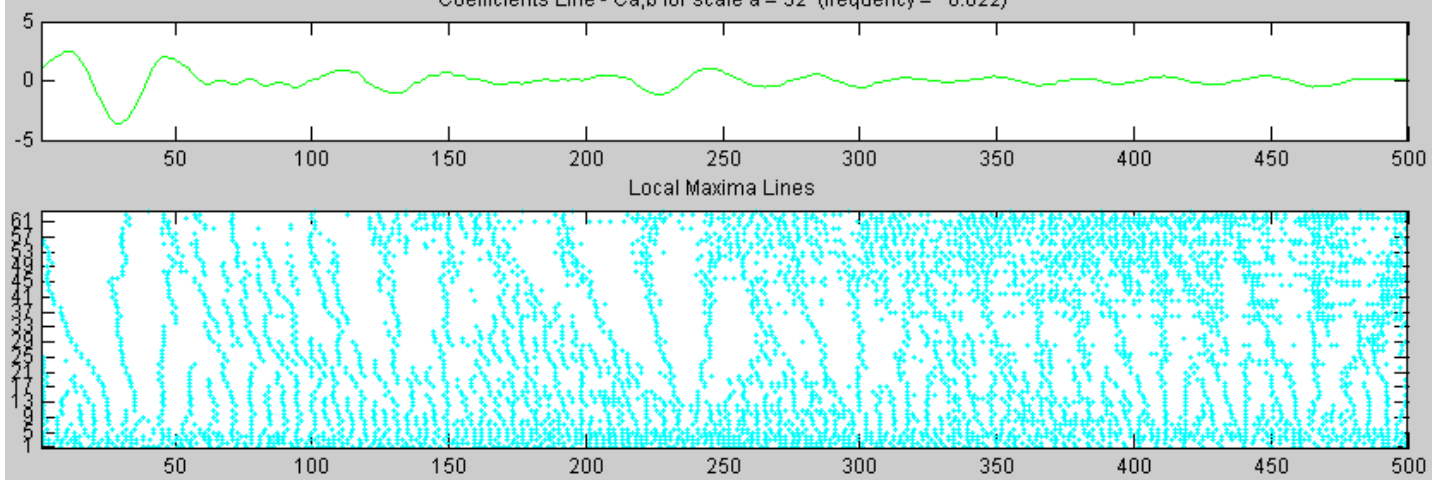

(b)

Figure 3. Improved wavelet transform time-frequency Graphs of LFM signals. (a) SNR $=-5 \mathrm{~dB}$. (b) SNR $=5$ dB. 
signals will be studied in a systematic, comprehensive and in-depth way, and the optimal corresponding intra-pulse feature extraction method are expected in the multidimensional transformation domain.

\section{Acknowledgements}

This work is supported partly by National Natural Science Foundation of China under Grant No. 61301205 and No. 61571146, National Defense Based Science Research Program under Grant No. JCKY2013604B001. This paper is funded by the International Exchange Program of Harbin Engineering University for Innovation-oriented Talents Cultivation.

\section{References}

[1] Stove, A.G., Hume, A.L. and Baker, C.J. (2004) Low Probability of Intercept Radar Strategies. IEEE Proceedings-Radar, Sonar and Navigation, 151, 249-260. https://doi.org/10.1049/ip-rsn:20041056

[2] Liu, F., Xu, H. and Tao, R. (2012) Research on Resolution between Multi-Component LFM Signal in the Fractional Domain. Science China Information Sciences, 55, 1301-1312. https://doi.org/10.1007/s11432-011-4324-6

[3] Wang, K., Jiang, Q.-X., Liu, F.-Z. and Pan, J.-F. (2017) A Design of Broad Band Multidimensional Radar Signal. Journal of Naval University of Engineering, 29, 3540.

[4] Moraitakis, I. and Fargues, M.P. (2000) Feature Extraction of Intra-Pulse Modulated Signals Using Time-Frequency Analysis. Proceedings of 21 st Century Military Communications Conference, Los Angeles, 22-25 October 2000, 737-741. https://doi.org/10.1109/MILCOM.2000.904028

[5] Czerwinski, P.N. (2007) Adaptive Short-Time Fourier Analysis. IEEE Signal Processing Letters, 4, 42-45. https://doi.org/10.1109/97.554468

[6] Poyil, A.T. and Nasimudeen, K.M. (2012) Multi Resolution Signal Analysis Using Improved Wigner Ville Distribution. 2012 International Conference on Communication, Information \& Computing Technology (ICCICT), Mumbai, 19-20 October 2012, 1-4. https://doi.org/10.1109/ICCICT.2012.6398197

[7] Ji, Z.H. and Yan, S.G. (2016) Improved Morlet Wavelet and Its Complete Transform. Computer Engineering and Applications, 52, 12-18.

[8] Yang, H.F. and He, Z.R. (2016) Time-Frequency Analysis in Radar Signal Recognition. Electronic Sci. \& Tech, 29, 58-61.

[9] Zhang, X.R. and Xie, H. (2013) A Novel Method Based on Time-Frequency Atom for Complex Modulated Radar Signal Sorting in Low SNR Condition. Applied Science and Technology, 40, 51-57.

[10] Kwok, H.K. and Jones, D.L. (2000) Improved Instantaneous Frequency Estimation Using an Adaptive Short-Time Fourier Transform. IEEE Transactions on Signal Processing, 48, 2964-2972. https://doi.org/10.1109/78.869059

[11] Khalil, N.H. (2007) Wavelet Analysis of Instantaneous Correlations with Application to Frequency Hopped Signals. Naval Postgraduate School, Monterey.

[12] Wei, K.-Y., Feng, L., Lin, X. and Liu, H.-J. (2017) Sorting Method of Radar Pulse Signal Based on Wavelet Analysis. Information Technology, 2017, 29-31. 
Submit or recommend next manuscript to SCIRP and we will provide best service for you:

Accepting pre-submission inquiries through Email, Facebook, LinkedIn, Twitter, etc. A wide selection of journals (inclusive of 9 subjects, more than 200 journals)

Providing 24-hour high-quality service

User-friendly online submission system

Fair and swift peer-review system

Efficient typesetting and proofreading procedure

Display of the result of downloads and visits, as well as the number of cited articles Maximum dissemination of your research work

Submit your manuscript at: http://papersubmission.scirp.org/

Or contact ijcns@scirp.org 